\title{
A Study and Evaluation of Transform Domain based Image Fusion Techniques for Visual Sensor Networks
}

\author{
Chaahat Gupta \\ Astt.Prof, CSE Deptt. \\ MIET,Jammu
}

\author{
Preeti Gupta
}

\begin{abstract}
This paper presents an evaluation of different image fusion techniques. There are many image fusion techniques which have been developed in a number of applications. Image fusion incorporates the data from several images of one scene to obtain an enlightening image which is more appropriate for human visual perception or additional vision processing. Image quality is closely connected to image focus. Image fusion has become one of the most recent and popular methods in the field of image processing. The discrete cosine transforms (DCT) based methods of image fusion are more suitable for energy consumption and time-saving in real-time systems using DCT based standards of still image.
\end{abstract}

\section{Keywords}

Image Fusion, Discrete Wavelet Transform, Discrete Cosine Transformations, Wavelet Transformations, Laplacian Pyramid, Visual Sensor Networks.

\section{INTRODUCTION}

Image fusion is a process which aims to merge important and relevant information from two or more images into a single image [1]. The outcome image will contain all the vital information as compare to input images. The new image will extract all the information from source images. Image fusion is a useful process for merging the single sensor and multisensor images to enhance the information [4]. The aim of image fusion is to merge information from multiple images in order to create an image that conveys only the useful information. Image fusion can process the images obtained from different sensors by a specific algorithm so that the resultant image is more reliable. The discrete cosine transforms (DCT) based method of image fusion is more relevant and time-saving in real-time systems. A more competent approach for fusion of multi-focus images depends on variance calculated in Discrete Cosine Transform. All sensors can obtain, generate and transfer data in every sensor networks [16]. Visual Sensor Networks refers to a system with a large number of cameras that are used to geographically expand the resources and monitoring of various points [5]. In VSN, sensors are cameras which can record either images or various video sequences.

\subsection{Levels of Image Fusion}

There are three levels of fusion which are given below:

a) Pixel level fusion- Pixel level fusion is the combination of the raw data from multiple source images into a single image.

b) Feature level fusion- Feature level fusion requires the extraction of dissimilar significant features from the source data before features are fused together.

c) Decision level fusion- Decision level fusion combines the results from multiple algorithms to yield a final fused decision.

\subsection{Image Fusion Techniques}

In the image fusion methods the good and information from each of the given images is fused together to form an output image whose quality is better than to any of the input images [6]. Image fusion methods can be classified into two methods as follow:

\section{- Spatial domain fusion method \\ - Transform domain fusion method.}

In spatial domain techniques, we directly deal with the pixel value of an image [3]. The various pixel values are manipulated to achieve the required result. For frequency domain methods, the pixel value is first changed into domain methods by applying discrete cosine transform (DCT) and discrete fourier transform (DFT) based fusion methods and rest of the image is enhanced by changing frequency component of an image. Figure 1 shows the fused image in 1(c) formed by image 1(a) and image 1(b).

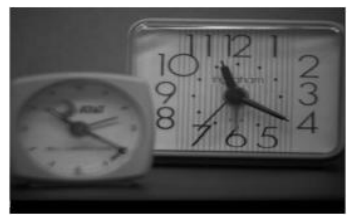

a) Left blurred image

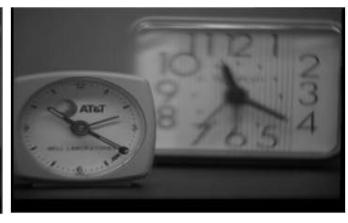

b) Right blurred image

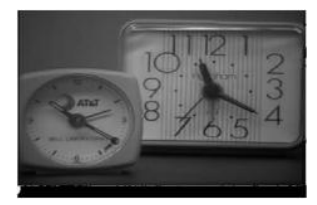

c) Fused Image

Figure 1: Fused image in figure 1(c) using images 1(a) and $1(b)$

\section{2. SPATIAL DOMAIN FUSION}

The various types of spatial domain methods are discussed in the following sections.

\subsection{Averaging based Fusion Method}

It is a known fact that regions of images that are in focus tend to be of higher pixel intensity. Therefore, averaging based fusion method is an easy way to achieve an output image with all regions in focus. The value of the pixel $P(i, j)$ of an image is taken and then added. The sum obtained is then divided by 2 to achieve the average. The average value is assigned to the corresponding pixel of the output image which is given in equation (1). This is repeated for all pixel values. 


$$
\mathrm{K}(\mathrm{i}, \mathrm{j})=\{\mathrm{X}(\mathrm{i}, \mathrm{j})+\mathrm{Y}(\mathrm{i}, \mathrm{j})\} / 2
$$

Where $X(i, j)$ and $Y(i, j)$ are two input images.

\subsection{Principle Component Analysis (PCA) based Fusion Method}

Principal component analysis provides a powerful tool for data analysis and pattern recognition which is used in image processing as a technique for data dimension reduction or their decorrelation of variables and data compression as well. Principal component analysis based fusion method is appropriate when measures on a number of observed variables have been obtained and further it is desired to develop a smaller number of artificial variables (called principal components) that will account for most of the variance in the experimental variables [17]. The PCA involve a mathematical formula that transforms a number of correlated variables into a number of uncorrelated variables called principal components. The Principal Component analysis method is used largely in image classification and image compression. It calculates a compact and optimal depiction of the data sets. The first principal component gives description for as much of the variance in the data as possible. The first principal component is taken along with the maximum variance. The second principal component points the direction of maximum variance as perpendicular to the first. The third principal component is taken along the direction of maximum variance in the subspace perpendicular to the first two and so on [19].

The various steps which are used in Principal Components Analysis based image fusion method are as follows and figure 2 depict Principal Components Analysis based image fusion method:-

1. The various images are formatted so that size of lowresolution multi-spectral images is the same as that of high resolution images.

2. PCA method transforms low resolution multi-spectral images to the principal component images.

3. The first principal component of an image is replaced with the high resolution image which is stretched to have almost the same variance and mean as the first principal component of an image.

4. The results of the high-resolution PAN data replace the first principal component image before the data is back transformed into the original space by PCA inverse transformation.

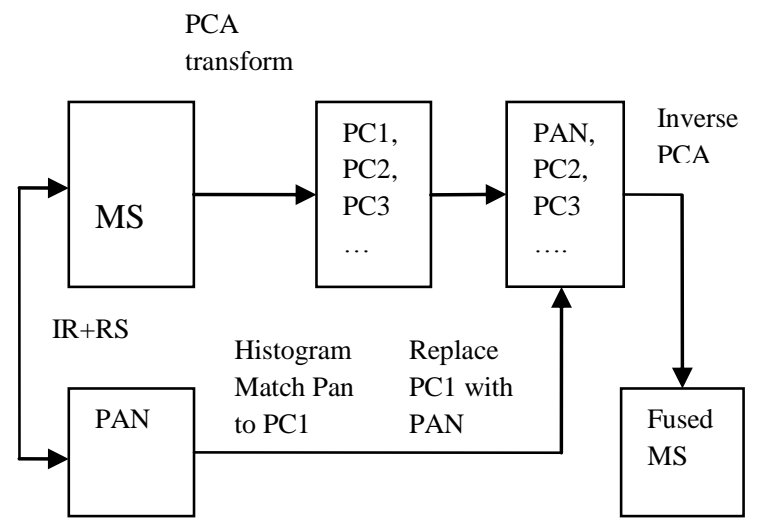

Figure 2: Principal Components Analysis based image fusion method

\section{TRANSFORM DOMAIN FUSION}

In frequency domain methods, the pixel value is first transferred in to domain methods by applying DCT and DWT based fusion ways and further image is enhanced by altering frequency component of an image. The following section discusses various fusion methods

\subsection{Laplacian Pyramid Fusion Method}

The basic idea behind the Laplacian pyramid is to perform a pyramid decomposition on every source image, then integrate of these decompositions to make a composite representation and finally reconstruct the fused image by performing an inverse pyramid transform [14]. The various steps used in Laplacian pyramid based fusion method are as follows:

1. The first step is to construct a pyramid for each source image.

2. Then the fusion is implemented at each level of the pyramid using a feature selection decision method.

3. The feature selection method selects the most significant pattern from the source image and copies it to the composite pyramid.

4. Finally, fused image is obtained by performing an inverse pyramid transform.

\subsection{Discrete Cosine Transform (DCT)}

Spatial domain image fusion methods are complicated and time consuming which are difficult to be performed on realtime images. Also, fusion approaches which are applied in DCT are very adept when the source images are coded in Joint Photographic Experts Group (JPEG) format or when the fused image will be saved or transmitted in JPEG format [9]. To perform the JPEG coding, an image is first subdivided into blocks of $8 \times 8$ pixels. The Discrete Cosine Transform (DCT) is then performed on every block. This generates 64 coefficients which are then quantized to reduce their magnitude [11]. The coefficients are then reordered into a one-dimensional array in a zigzag manner before further entropy encoding takes place. The compression is achieved in two stages the first is during quantization and the second during the entropy coding procedure. JPEG decoding is the reverse process of encoding [13].

\subsection{Discrete Wavelet Transform (DWT)}

The wavelet transform decomposes the image into low-low, low-high, high-low and high-high spatial frequency bands at different scales [2][10]. The LL band contains the approximation coefficients whereas the other bands contain directional information due to spatial orientation. $\mathrm{LH}$ band contains the horizontal detail coefficients. HL band contains the vertical detail coefficients. HH contains the diagonal detail coefficients and also contain the higher absolute values of wavelet coefficients correspond to salient features such as edges or lines [7][12]. Figure 3 shows Discrete Wavelet Transform (DWT) based image fusion. The wavelets-based approach performs the following tasks:-

1. It is a multi scale (multi resolution) approach well suited to manage the different image resolutions. It is Useful in a number of image processing applications including the image fusion [15].

2. The discrete wavelets transform (DWT) allows the image decomposition in different kinds of coefficients preserving the image information. 
3. Such coefficients approaching from different images can be appropriately combined to obtain new coefficients so that the information in the original images is collected appropriately.

4. After the coefficients are merged then the final fused image is achieved by applying the inverse discrete wavelets transform (IDWT), where the information in the merged coefficients is also preserved.

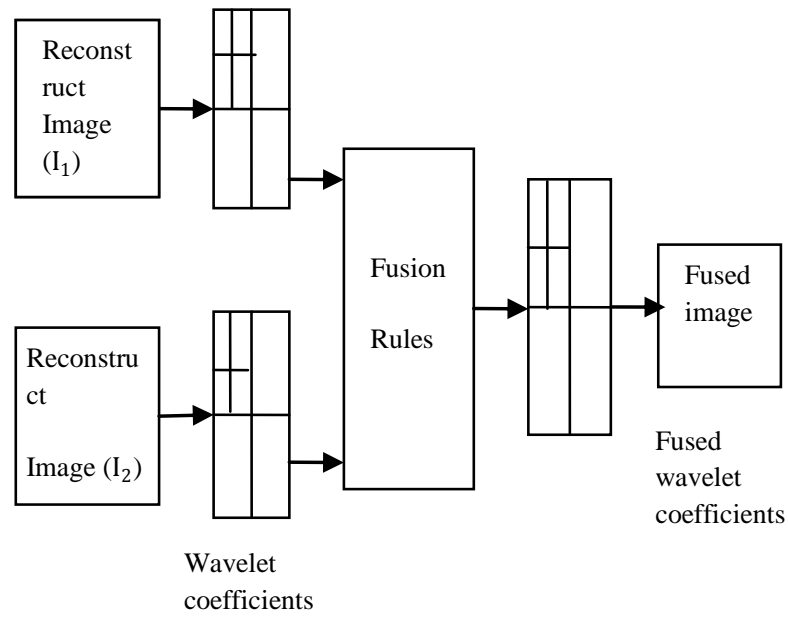
Figure 3: Discrete Wavelet Transform (DWT) based
Image Fusion

\section{LITERATURE SURVEY}

Image Fusion is used effectively in image processing field. Different image fusion methods have been proposed in the literature survey. Image fusion enhances the quality of image by removing the blurriness and the noise of the images. Image fusion takes place at three different levels i.e. pixel, feature and decision. Image fusion methods can be broadly classified into two categories, i.e., spatial domain fusion and transform domain fusion. Averaging, Brovey method and Principal Component Analysis (PCA) based methods are spatial domain methods. But the problem with spatial domain methods is that they produce distortion in the fused image. This limitation is overcome by introduction of transform domain approach. A brief summary of the literature is given below as follow:

Xiangzhi Bai et al. (2011) have presented the toggle contrast operator in image fusion for edge preserving and image sharpening. The operator used structuring elements with similar shape and expands sizes in the beginning. After that, the dilation and erosion characteristics extracted the edge data of actual image and final dilation and erosion are constructed through the pixel wise maximum operation at all scales .Finaly, the output image of fusion is formed by joining the final dilation and erosion into base image compute from actual image.

Li et al. (2008) have examined image fusion by merging of curvelet and wavelet transform. Each of input images is broken down by curvelet transform and after that wavelet transform method coefficients is fused. It was observed that by applying inverse curvelet transform fused image was restored. This method had superior performance to other methods. The limitation of this system was that it took more time than wavelet transform because of two non-identical multi-scale decomposition processes.
Li et al. (2013) have analyzed region-based image fusion method with Local Spatial Frequency (LSF). LSF for each pixel of input images is evaluated first and then segmentation of the input images is done with the average image. Then label the input image and shared region representation is obtained from segmented image. Region spatial frequency is used to guide the fusion process and recognition of major features in shared region representation. By using this method, authors discovered that maximum detail information can be shifted from input images to fused image. Moreover, it was studied that this algorithm has many advantages of preserving detail information.

Wang et al. (2013) have explored multi-spectral image fusion method based on the features of imaging system. It was proposed that in the beginning the method performs multiresolution decomposition to multi-spectral and panchromatic images adopting with non sub sample contour transform. After that, the panchromatic image injection model is created by examining the characteristics of image system of multispectral image in detail and finally, the detail data of panchromatic image to every spectrum to multi-spectral image is injected by this method. The final image obtained by decomposition coefficient is reconstructed by inverse non subsample contour transform. It was discovered that, this method not only gave the details of panchromatic image better but also gave good results for spectral features of multi-spectral images by minimizing the spectrum distortion problem efficiently.

Galande et al. (2013) have reviewed the medical image fusion to enhance the content of fusing images taken from non-identical imaging tools like magnetic resonance imaging (MRI), computer tomography (CT) etc. In this paper, image fusion based is based on two categories. First is the pixel level image fusion and second is transformed based image fusion. It was discovered that it was very tough to select only one method for all applications. But then it was inferred that from visual perspective, fuzzy inference system gave better results in fused images. Also the authors analyzed that it was not possible to design universal image fusion method which is applicable to all fusion algorithms.

Garg et al. (2014) have reviewed the different types of multimodal image fusion algorithms which uses non-identical focus measure based on morphological opening and closing spatial frequency. The performance of these methods is examined to know how focused regions in images are calculated to obtain a fused image. The problem of fixed size block is solved by using quad-tree structure and genetic algorithm. It was found that Hybrid technique using frequency domain and spatial domain can be used to get better results.

Hui et al. (1995) have presented in there paper, a wavelet transform based fusion method. In this method, DWT is applied on source images, then all the wavelet coefficients are combined and the fused image is achieved by applying the inverse wavelet transform based method. An area based maximum selection rule and a consistency verification steps are used for feature selection.

Lixin, ET al. (2013) have discussed the wavelet-based method for multi-focus image fusion in their paper. It was proposed that first the pending images are decomposed by using wavelet method into four bands i.e., LL, LH, HL and $\mathrm{HH}$ and then sub band $\mathrm{LH}, \mathrm{HL}$ and $\mathrm{HH}$ are synthesized to get three directions of high-frequency details of images. The authors studied the enhancement of the fused image with no 
artificial effect or blocking effect. The proposed method is better in terms that it has faster speed, it consumes less memory and has ease of implementation. This method also tells that the visual impression and fused image more suitable for human visual observation

Haghighat et al. (2011) have proposed a new DCT based fusion technique for multi-focus images. This fusion method was based on variance definition in DCT domain. The variance value is calculated from all DCT coefficients which is used as a contrast criterion in image processing applications. The proposed method performs the better result as compared to previous DCT based methods both in quality and complexity reduction.

Singh et al. (2014) have presented the combination of DCT and adaptive histogram equalization in their study and have provided an enhancement over the existing based DCT based image fusion techniques. It was studied that image fusion based on DCT provide less PSNR value but maximum mean square error. Many researchers have ignored image filtering and restoration which is must require of image fusion. So this method combines DCT and adaptive histogram equalization to give better results than any other method.

Khare et al. (2013) have proposed a Biorthogonal wavelet transform (BWT) based image fusion method using absolute maximum fusion rule. Authors have suggested the adoption of this method because biorthogonal wavelet transform contains spline wavelets. With these spline wavelets symmetry and perfect reconstruction is possible using FIR (Finite Impulse Response) filters. The symmetry means that the filters have linear phase so that BWT is capable to preserve edge information and hence reduces the distortions in the fused image. The experimental results showed that BWT based fusion method gave better results and it is more applicable because it can retain information of individual image like edges, lines, curves and boundaries in the fused image in a better way as compare to traditional spatial domain based image fusion methods such as Linear fusion method, Principal Component Analysis based fusion and Sharpness criteria based image fusion .

Rockinger et al. (1997) has given a shift invariant wavelet transform based fusion method. In this proposed method, the fusion process should be shift invariant which means that it should not depend on location of object in an input image and it should be temporal stable and consistent within the input sequence.

Amutha et al. (2014) have proposed a simple and efficient DCT based image fusion technique. Authors have suggested the adoption of this method because DCT based fusion overcomes the computation and energy limitation of low power device. In this fusion method, the image blocks with higher value of $\mathrm{AC}$ coefficients are absorbed into the fused image. It was analyzed that it was extremely fast as it does not involve any complex floating point arithmetic operations like mean or variance calculation. The proposed fusion technique considerably reduces the computational complexity without compromising image quality.

\section{GAPS IN EARLIER WORK}

The literature review has shown that the most of the existing research has neglected the followings things:-

1. It has been concluded from the literature survey that most of the existing methods are based upon transformations thus leading to some color artefacts which may lower the performance of the transform based image fusion methods.

2. By studying the literature survey it has been observed that the problem of the uneven illuminate has also been overlooked in most of the existing works on fusion.

\section{CONCLUSION}

This paper has presented a related work on the different image fusion methods. The main aim of image fusion in multi-focus cameras is to merge the data or information from several pictures of the similar scene in order to deliver only the multi-focused image. It has been seen techniques based on DCT of image fusion are more suitable in aspect of energy saving and time-saving in real-time systems for still images or videos. In our paper, an adept technique for fusion of multi-focus images based on variance calculated in DCT domain has been also reviewed. We have concluded that existing research methods neglected the fact that most of existing methods are based upon transformations, thus, leading to some color artefacts which may lower the performance of the transform based image fusion methods. Also, we have found that the most of the existing literature has neglected the problem of noise which will be present in fused images due to integration of two images. So in near future, we will propose a new technique which will merge newly modified DCT and PCA to raise the quality of fusion of various images. In our present work, we have done no implementation so far. For future, we plan to use MATLAB tool for implementation of our proposed work.

\section{REFERENCES}

[1] Bai, Xiangzhi, Fugen Zhou, and Bindang Xue. "Edge preserved image fusion based on multiscale toggle contrast operator." Image and Vision Computing 29.12 (2011): 829-839.

[2] Li, Shutao, and Bin Yang. "Multifocus image fusion by combining curvelet and wavelet transform." Pattern Recognition Letters 29.9 (2008): 1295-1301.

[3] Li, Qingping, et al. "Region-based multi-focus image fusion using the local spatial frequency." Control and Decision Conference (CCDC), 2013 25th Chinese. IEEE, 2013.

[4] Wang, Jingling, et al. "Multi-spectral image fusion based on the characteristic of imaging system."International conference on Information and Automation, 2013.IEEE, 2013.

[5] Galande, Ashwini, and Ratna Patil. "The art of medical image fusion: A survey." Advances in Computing, Communications and Informatics (ICACCI), 2013 International Conference on. IEEE, 2013.

[6] Garg, Rishu, Preeti Gupta, and Harvinder Kaur. "Survey on multi-focus image fusion algorithms." Engineering and Computational Sciences (RAECS), 2014 Recent Advances in. IEEE, 2014.

[7] Li, Hui, B. S. Manjunath, and Sanjit K. Mitra. "Multisensor image fusion using the wavelet transforms." Graphical models and image processing, vol. 3, pp. 235-245. IEEE, 1997.

[8] Liu, Lixin et al. "An Effective Wavelet-based Scheme for Multi-focus Image Fusion ."Mechatronics and Automation, 2013 Takamatsu, Japan.IEEE, 2013.

[9] Haghighat, Mohammad Bagher Akbari, Ali 
Aghagolzadeh, and Hadi Seyedarabi. "Multi-focus image fusion for visual sensor networks in DCT domain."Computers \& Electrical Engineering 37.5 (2011): 789-797.

[10] Om Prakash, Richa Srivastava, Ashish Khare. "Biorthogonal Wavelet Transform Based Image Fusion Using Absolute Maximum Fusion Rule." In Image processing, 2013 International Conference on Information and Communication Technologies, pp. 577 582. IEEE, 2013

[11] Singh, Jagdeep, and Vijay Kumar Banga. "An Enhanced DCT based Image Fusion using Adaptive Histogram Equalization." International Journal of Computer Applications 87.12 (2014): 26-32.

[12] O.Rockinger. "Image sequence fusions using a shiftinvariant wavelet transform." In image processing, 1997 International Conference on, vol. 3, pp. 288-291. IEEE, 1997.

[13] Y.AsnathVictyPhamila, R.Amutha. "Discrete Cosine Transform based fusion of multi-focus images for visual sensor networks." In Signal Processing, 2013 International Conference on, pp.161-170. IEEE, 2013.

[14] Wang, Wencheng, and Faliang Chang. "A multi-focus image fusion method based on Laplacian pyramid." Journal of Computers 6.12 (2011): 2559-
2566 .

[15] Ali, Syed Twareque, Jean-Pierre Antoine, and JeanPierre Gazeau. "Discrete Wavelet Transforms." Coherent States, Wavelets, and Their Generalizations. Springer New York, 2014. 379-410.

[16] Haghighat, Mohammad Bagher Akbari, Ali Aghagolzadeh, and Hadi Seyedarabi. "Real-time fusion of multi-focus images for visual sensor networks." Machine Vision and Image Processing (MVIP), 2010 6th Iranian. IEEE, 2010.

[17] Patil, U.; Mudengudi, U., "Image fusion using hierarchical PCA.," Image Information Processing (ICIIP), 2011 International Conference on, pp.1-6, 2011.

[18] Wen Cao, Bicheng Li, Yong Zhang, "A remote sensing image fusion method based on PCA transform and wavelet packet transform," Neural Networks and Signal Processing, 2003. Proceedings of the 2003 International Conference on , Vol.2, pp.976-981, 2003.

[19] Mahajan Rajiv, Bamba Sheffali, "Performance Evaluation of Modified Color Based Edge Detection of Remote Sensing Images Using Fuzzy Logic," International Journal of Advanced Research in Computer Science and Software Engineering, Volume 4, Issue 8, August 2014,pp.334-343. 